\title{
Una comparación entre los estudios de fase 3 \\ $y$ un estudio de fase 4 de nalmefeno en el trastorno por uso de alcohol. ¿Existen diferencias?
}

\section{A comparison between phase-III trials and a phase-IV study of nalmefene in alcohol use disorder patients. Is there a difference?}

\author{
Pablo Barrio*, Lluisa Ortega*, Josep Guardia**, Carlos Roncero***, \\ Lara Yuguero****, Antoni Gual*. \\ * Unidad de Conductas Adictivas, Hospital Clínic, Universidad de Barcelona, Barcelona, España. ** Unidad de Conductas \\ Adictivas, Departamento de Psiquiatría, Hospital de la Santa Creu i Sant Pau, Barcelona, España. *** Complejo Asistencial \\ Universitario de Salamanca. Instituto de Biomedicina de Salamanca, Universidad de Salamanca. **** Unidad de Conductas \\ Adictivas, Germanes Hospitalàries, Sant Boi, Barcelona, España.
}

\section{Resumen}

En muchas disciplinas médicas existen diferencias significativas entre las muestras procedentes de estudios experimentales y las muestras procedentes de ámbitos clínicos, como es por ejemplo el caso de la hipertensión. El objetivo del presente estudio fue comparar las muestras procedentes de los ensayos pivotales de fase 3 de nalmefeno con la muestra de un estudio de fase 4 realizado recientemente. Las características basales de las muestras se compararon mediante técnicas univariantes. Se encontraron diferencias significativas entre el porcentaje de los participantes consumidores de alcohol de bajo riesgo. También se encontraron diferencias en los patrones de prescripción y en la toma de nalmefeno, así como en el porcentaje de comorbilidades psiquiátricas y adictivas, que fueron muy superiores en el estudio de fase 4. En su conjunto, estos datos sugieren que en el campo del trastorno por uso de alcohol (TUA) existen también diferencias relevantes entre muestras procedentes de estudios experimentales y muestras procedentes de estudios clínico-observacionales. Este hecho refuerza la necesidad de que los estudios de fase 3 sean complementados con estudios observacionales de fase 4 .

Palabras clave: Fase 3; Fase 4; Validez externa; Nalmefeno; Trastorno por uso de alcohol.

\begin{abstract}
Concerns regarding the external validity of phase-III trials are common to many medical disciplines, with relevant discrepancies found between experimental and clinical samples in some diseases such as hypertension. The aim of this study was to compare the samples included in the pivotal, phase-III clinical trials of nalmefene with that of a recently conducted phase-IV trial. Baseline characteristics of the studies were compared through univariate analysis. Significant differences were found in the percentage of low-risk drinkers included. Differences were also found in the prescription and intake pattern of nalmefene, as well as in the rate of psychiatric and addictive comorbidities, which were much higher in the phase-IV study. These data suggest that in the field of alcohol use disorders there are also relevant differences between experimental and clinical samples, a fact that reinforces the need for phase-III trials to be balanced with observational, phase-IV trials.
\end{abstract}

Keywords: Phase-III trial; Phase-IV trial; External validity; Nalmefene; Alcohol use disorders. 
$\mathrm{E}$ 1 alcohol supone una carga considerable en las sociedades de todo el mundo, la mayoría de la cual es infligida por las personas que lo consumen en grandes cantidades: aquellas afectadas por un trastorno por uso de alcohol (TUA) (Whiteford et al., 2013). Por lo tanto, el tratamiento de los TUA tiene una importancia alta. La recomendación habitual combina estrategias psicosociales y farmacológicas. Han aparecido compuestos diferentes para el tratamiento farmacológico en décadas recientes, con eficacia probada en varios resultados. Tal es el caso de nalmefeno, un opioide antagonista aprobado recientemente para el tratamiento de pacientes alcohólicos cuyo objetivo es conseguir una reducción objetiva. Dicha aprobación se basó en tres ensayos pivotales de fase 3 (Gual et al., 2013; Mann, Bladström, Torup, Gual y van den Brink, 2013; van den Brink et al., 2013).

Los ensayos de fase 3 son un paso básico y muy importante en el proceso de aprobación de un medicamento por parte de las agencias reguladoras. Suelen ser ensayos de gran tamaño y aleatorios con una muestra de estudio homogénea. Todas estas características resultan en un poder estadístico alto y una validez interna alta.

Mientras que los diseños experimentales como estudios de fase 3 son críticos para valorar la validez interna, y son la piedra angular de la evaluación de la eficacia de un medicamento, en muchas áreas se ha subrayado extensamente que lo habitual es que la validez externa quede descuidada de manera desproporcionada (Dekkers, von Elm, Algra, Romijn y Vandenbroucke, 2010; Pearson y Coomber, 2010; Rothwell, 2005). Este hecho podría aportar consecuencias relevantes, tales como el hecho que los pacientes de entornos experimentales podrían tener diferencias significativas en comparación con los pacientes de entornos clínicos (Hoertel et al., 2014; Uijen, Bakx, Mokkink y van Weel, 2007), en último término arriesgando la fiabilidad, aplicabilidad e incluso la relevancia de los hallazgos experimentales (Persaud y Mamdani, 2006).

En este contexto, estudios previos en varias enfermedades, tales como la hipertensión, ansiedad social, artritis reumatoide y otras (Farahani, Levine, Gaebel y Thabane, 2005), han encontrado que no siempre hay similitud entre los pacientes de fase 3 y fase 4 , y esto puede conllevar implicaciones relevantes. Por lo tanto, pensamos que es necesario evaluar, en el ámbito de los TUA, si los pacientes de la "vida real" son comparables a los pacientes de estudios experimentales previos, y cuáles pueden ser las diferencias existentes. De hecho, este es uno de los motivos que llevan a la necesidad de realizar estudios de fase 4 (Linden, 1984).

El propósito de este manuscrito es comparar las características basales de pacientes participantes en un ensayo de fase 4 de nalmefeno con los pacientes que participaron en los ensayos de fase 3 .

\section{Método}

Este estudio comparó los pacientes de dos tipos de estudios diferentes. El primer grupo de pacientes era de un estudio de fase 4 observacional, multicéntrico y de brazo único que se realizó entre pacientes ambulatorios con TUA que tomaban nalmefeno por primera vez como tratamiento de su trastorno. Para participar en este ensayo de fase 4, los pacientes tenían que ser adultos (edad $\geq 18$ años), estar diagnosticados con dependencia del alcohol según los criterios del Manual Diagnóstico y Estadístico de los Trastornos Mentales (DSM-IV TR) o la Clasificación Internacional de Enfermedades (ICD-10) y que, según criterios habituales en la práctica clínica, habían comenzado a usar nalmefeno por primera vez. El estudio consistió en 4 visitas: basal, a 1 mes, a los 6 meses y a los 12 meses. Los pacientes continuaron con su tratamiento habitual independientemente de las visitas y los procedimientos del estudio.

El segundo grupo estaba compuesto de pacientes que habían participado en los ensayos de fase 3 de nalmefeno. Se recopilaron datos de estos pacientes de publicaciones existentes en la literatura. Los ensayos de fase 3 se analizaron de forma independiente con el fin de realizar comparaciones. Por lo tanto, se crearon 4 grupos en total. Cada ensayo de fase 3 se comparó con el estudio de fase 4 . Se incluyeron todos los variables basales disponibles para los 4 estudios. Las comparaciones estadísticas se hicieron mediante análisis univariantes (prueba t o de chi cuadrado, según el tipo de variable).

\section{Resultados}

Se incluyeron 110 pacientes en total de 4 centros de la región española de Cataluña entre 2015 y 2016. Los resultados completos del estudio de fase 4 están disponibles en otra publicación (Barrio, Ortega, Guardia, Roncero, Yuguero y Gual, 2018). La tabla 1 muestras las diferencias entre este estudio y el ensayo de fase 3 de nalmefeno respecto de los variables basales del estudio principal.

Hubo diferencias importantes en cuanto al porcentaje de nivel de riesgo bajo de consumo de alcohol en comparación con este estudio que mostró una tasa mucho más elevada (45\%) respecto de los ensayos ESENSE (1-5\%). En consecuencia, en las categorías de riesgo alto/muy alto, los números se invirtieron, con el $38 \%$ de los pacientes de este estudio categorizados aquí, comparado con el 76-78\% de los ensayos de fase 3. Otro ejemplo de estas diferencias relevantes es el hecho que, en este estudio, el consumo medio de alcohol (60.4 g/día) fue significativamente más bajo (85-92 g/día en los ensayos ESENSE).

También relevante, encontramos una prevalencia significativamente menor de una historia familiar de problemas relacionados con el alcohol en nuestra muestra. Finalmente, observamos una proporción más elevada en el ensayo de fase 4 del porcentaje de días de ingesta de medicación 
durante el estudio. También es relevante que fue más alto el número de pacientes que tomaban nalmefeno diariamente. Dado el criterio de inclusión de ensayos de fase 3, se hallaron comorbilidades adictivas únicamente en el estudio de fase 4. De manera similar, sólo el ensayo SENSE permitió comorbilidades psiquiátricas entre los pacientes, pero a pesar de esto, su frecuencia era mucho menor que en el estudio de fase 4.

\section{Discusión}

Este estudio halló diferencias significativas entre pacientes de fase 4 y de fase 3 que tomaban nalmefeno. En comparación con los ensayos de fase 3 , los pacientes de entornos reales mostraron una tasa más alta de comorbilidades adictivas y psiquiátricas, un contraste obvio con las muestras aleatorias y controladas de estudios con criterios de inclusión habitualmente más restrictivos. De hecho, la tasa alta de comorbilidades psiquiátricas en pacientes con TUA es un fenómeno claramente documentado (Fein, 2015; Flensborg-Madsen et al., 2009). Este hallazgo apoya las críticas respecto de la validez externa de estudios experimentales (Persaud y Mamdani, 2006) y, a la vez, sugiere que estudios como el SENSE (van den Brink et al., 2014), que incluyen a pacientes con comorbilidades psiquiátricas, deberían ser la norma en lugar de la excepción.

Hemos de destacar, además, que gran parte de los pacientes en el ensayo de fase 4 ya estaban clasificados en la línea basal como consumidores de alcohol de bajo riesgo. Es más, casi la mitad de los pacientes eran considerados consumidores de alcohol de bajo riesgo al inicio del estudio, un hecho que a primera vista puede parecer contradictorio a las indicaciones terapéuticas de nalmefeno. No obstante, hemos de destacar que la valoración de riesgo para los fines del estudio de fase 4 se basó en los 28 días anteriores. Es probable que los profesionales clínicos tengan en cuenta un periodo temporal más amplio al categorizar el riesgo de sus pacientes. Además, de manera similar a lo observado en los ensayos de fase 3 , es posible que el mero hecho de decidir acogerse a un tratamiento resulte en reducciones importantes del consumo de alcohol por parte de los pacientes. Curiosamente, el porcentaje de consumidores de alcohol de bajo riesgo en el ensayo de fase 4 es muy similar a la de los estudios de fase 3 si sumamos los consumidores de alcohol de bajo riesgo basales y los consumidores de alcohol de bajo riesgo antes del inicio de su tratamiento con nalmefeno.

La edad de inicio de problemas relacionados con el alcohol fue otro variable que mostró diferencias claras.

Tabla 1. Características sociodemográficas principales de los pacientes participantes y comparación con los estudios de fase 3

\begin{tabular}{|c|c|c|c|c|}
\hline Característica & Fase $4(n=110)$ & $\begin{array}{l}\text { ESENSE } 1 \\
(\mathrm{n}=306)\end{array}$ & $\begin{array}{l}\text { ESENSE } 2 \\
(\mathrm{n}=358)\end{array}$ & $\begin{array}{c}\text { SENSE } \\
(n=509)\end{array}$ \\
\hline Edad: media $(S D)$ & $44,4(9,4)$ & $51,0(10,1)^{*}$ & $45,1(10,7]$ & $44,3(11,2]$ \\
\hline Estudios superiores (\%) & $27,3 \%$ & & & $32,4 \%$ \\
\hline Edad al inicio de los problemas relacionados con el alcohol: media (SD) & $23(12,4)$ & $37,9(13,1)^{*}$ & $32,6(10,8)^{*}$ & $33,4(11,6)^{\star}$ \\
\hline \multicolumn{5}{|l|}{ Nivel de riesgo de consumo de alcohol } \\
\hline Bajo (\%) & $45,5 \%$ & $3 \% *$ & $1,4 \% *$ & $15,5 \%$ * \\
\hline Medio (\%) & $16,4 \%$ & $22,2 \%$ & $19,0 \%$ & $32,8 \% *$ \\
\hline Alto (\%) & $21,8 \%$ & $37,3 \% *$ & $36,0 \% *$ & $29,1 \%$ \\
\hline Muy Alto (\%) & $16,3 \%$ & $39,9 \% *$ & $43,6 \% *$ & $22,4 \%$ \\
\hline Alanina Aminotransferasa (IU/L): media (SD) & $29,2(15,5)$ & 29,2 & 28,7 & 28,5 \\
\hline Tratamientos anteriores para dependencia del alcohol (\%) & $46,4 \%$ & $29,7 \% *$ & $39,7 \%$ & $33,6 \% *$ \\
\hline Tratamientos anteriores para síndrome de abstinencia del alcohol (\%) & $30 \%$ & $19,6 \% *$ & $15,9 \% *$ & $26,9 \%$ \\
\hline Historial personal de trastornos psiquiátricos (\%) & $36,4 \%$ & & & $3,7 \% *$ \\
\hline Historial familiar de problemas relacionados con el alcohol (\%) & $48,2 \%$ & $62,4 \% *$ & $60,1 \% *$ & $51,7 \%$ \\
\hline Comorbilidades adictivas*** (en el pasado o en la actualidad) (\%) & $65,5 \%$ & & & \\
\hline Porcentaje de días de ingesta de medicación del estudio (\%) & $64 \%$ & $48 \% *$ & $57 \%$ & $48,4 \% *$ \\
\hline Días mensuales de consumo alto de alcohol (línea basal): media (SD) & $13,5(11)$ & $19,5(7,3) *$ & $19,7(7,0)^{*}$ & $14,1(6,2)$ \\
\hline Consumo medio de alcohol (gramos diarios; línea basal): media (SD) & $60,4(74,6)$ & $84,8(42,1) *$ & $92,2(46,9)^{*}$ & $68,6(40,0)$ \\
\hline
\end{tabular}

Nota. ${ }^{*}=$ significativo a $\mathrm{p}<, 05$ cuando comparado con los valores del estudio de fase IV usando pruebas monofactoriales (t de Student o chi cuadrado).

** definido como la presencia de diabetes, hipertensión, colesterol sanguíneo alto o cualquier otra condición médica de importancia.

*** definido como cualquier trastorno de uso de sustancia (exceptuando la dependencia de la nicotina), en el pasado o en la actualidad, según la valoración clínica en la primera visita como parte de este estudio. 
Aunque es de esperar que haya diferencias reales entre la vida real y los estudios experimentales, también pueden deberse al uso de métodos de recolección distintos. Mientras que una menor edad de inicio de problemas relacionados con el alcohol puede sugerir mayor severidad de la enfermedad, los parámetros basales de alcohol fueron, al contrario, más leves en la muestra del estudio de fase 4, con menos días de consumo de alcohol alto y un consumo de alcohol medio más bajo. Aparentemente, los pacientes en el ensayo de fase 4 tenían una tasa superior de ingesta de medicación. De hecho, los resultados de la fase 4 (Barrio et al., 2018) sugieren que, a pesar de estar etiquetada la medicación como de ingesta "según necesidad", en la práctica real los pacientes y los profesionales clínicos con frecuencia la usan de manera programada y a diario.

Este estudio tiene varias limitaciones. La más relevante es el diseño diferente de los ensayos de fase 4 y fase 3 , un hecho que implica cautela al interpretar las comparaciones hechas en este estudio. También, es importante mencionar que el ensayo de fase 4 incluyó a pacientes de 4 centros diferentes, todos en la misma ciudad. En este sentido, los ensayos de fase 3 tuvieron una representación mucho más amplia, con pacientes participantes de diferentes países.

\section{Conclusiones}

En conjunto, pensamos que este estudio sugiere, como se ha mostrado en otras enfermedades, que las muestras de estudios experimentales pueden diferir en varios aspectos de los pacientes en la práctica clínica rutinaria. Aunque han de fomentarse los esfuerzos centrados en aumentar la validez externa de los estudios de fase 3 , este estudio también confirma que los estudios de fase 4 son, sin duda, una parte crítica del proceso de investigación.

\section{Contribuciones}

Pablo Barrio y Antoni Gual diseñaron el estudio. Pablo Barrio redactó el borrador inicial del manuscrito. Los demás autores han contribuido hacia la edición y la revisión del mismo. Todos los autores aprobaron su versión definitiva.

\section{Financiación}

Lundbeck financió este estudio. Dicho sponsor participó en el diseño del estudio, pero no en la recolección y análisis de datos o en la redacción del manuscrito, ni en la decisión de presentar el manuscrito para su publicación.

\section{Conflicto de intereses}

Dr. Barrio, Dr. Roncero, Dr. Guardia y Dr. Gual han cobrado honorarios de Lundbeck. Dr. Barrio también ha cobrado honorarios de Pfizer. Dr. Roncero ha cobrado ho- norarios de Janssen-Cilag, Otsuka, Server, GSK, Rovi, Astra, MSD y Sanofi. Dr. Yuguero y Dr. Ortega declaran la inexistencia de conflicto de intereses.

\section{Reconocimientos}

Los autores desean expresar su gratitud a los participantes en el estudio.

\section{Referencias}

Barrio, P., Ortega, L., Guardia, J., Roncero, C., Yuguero, L. y Gual, A. (2018). Who Receives Nalmefene and How Does It Work in the Real World? A Single-Arm, Phase IV Study of Nalmefene in Alcohol Dependent Outpatients: Baseline and 1-Month Results. Clinical Drug Investigation, 38, 147-155. doi:10.1007/s40261-017-0590-4.

Dekkers, O. M., von Elm, E., Algra, A., Romijn, J. A. y Vandenbroucke, J. P. (2010). How to assess the external validity of therapeutic trials: a conceptual approach. International Journal of Epidemiology, 39, 89-94. doi:10.1093/ ije/dyp174.

Farahani, P., Levine, M., Gaebel, K. y Thabane, L. (2005). Clinical data gap between phase III clinical trials (pre-marketing) and phase IV (post-marketing) studies: evaluation of etanercept in rheumatoid arthritis. The $\mathrm{Ca}$ nadian Journal of Clinical Pharmacology, 12, e254-263.

Fein, G. (2015). Psychiatric Comorbidity in Alcohol Dependence. Neuropsychology Review, 25, 456-475. doi:10.1007/ s11065-015-9304-y.

Flensborg-Madsen, T., Mortensen, E. L., Knop, J., Becker, U., Sher, L. y Grønbæk, M. (2009). Comorbidity and temporal ordering of alcohol use disorders and other psychiatric disorders: results from a Danish register-based study. Comprehensive Psychiatry, 50, 307-314. doi:10.1016/j.comppsych.2008.09.003.

Gual, A., He, Y., Torup, L., van den Brink, W., Mann, K. y ESENSE 2 Study Group. (2013). A randomised, double-blind, placebo-controlled, efficacy study of nalmefene, as-needed use, in patients with alcohol dependence. European Neuropsychopharmacology, 23, 1432-1442. doi:10.1016/j.euroneuro.2013.02.006.

Hoertel, N., de Maricourt, P., Katz, J., Doukhan, R., Lavaud, P., Peyre, H. y Limosin, F. (2014). Are participants in pharmacological and psychotherapy treatment trials for social anxiety disorder representative of patients in real-life settings? Journal of Clinical Psychopharmacology, 34, 697-703. doi:10.1097/JCP.0000000000000204.

Linden, M. (1984). Phase-IV Research: Specifics, Objectives and Methodology. Pharmacopsychiatry, 17, 140-142. doi:10.1055/s-2007-1017425.

Mann, K., Bladström, A., Torup, L., Gual, A. y van den Brink, W. (2013). Extending the treatment options in alcohol dependence: a randomized controlled study of 
as-needed nalmefene. Biological Psychiatry, 73, 706-713. doi:10.1016/j.biopsych.2012.10.020.

Pearson, M. y Coomber, R. (2010). The challenge of external validity in policy-relevant systematic reviews: a case study from the field of substance misuse. Addiction, 105, 136-145. doi:10.1111/j.1360-0443.2009.02713.x.

Persaud, N. y Mamdani, M. M. (2006). External validity: the neglected dimension in evidence ranking. Journal of Evaluation in Clinical Practice, 12, 450-453. doi:10.1111/ j.1365-2753.2006.00730.x.

Rothwell, P. M. (2005). External validity of randomised controlled trials: "to whom do the results of this trial apply?”. Lancet, 365, 82-93. doi:10.1016/S01406736(04) 17670-8.

Uijen, A. A., Bakx, J. C., Mokkink, H. G. A. y van Weel, C. (2007). Hypertension patients participating in trials differ in many aspects from patients treated in general practices. Journal of Clinical Epidemiology, 60, 330-335. doi:10.1016/j.jclinepi.2006.05.015.

van den Brink, W., Aubin, H. J., Bladstrom, A., Torup, L., Gual, A. y Mann, K. (2013). Efficacy of as-needed nalmefene in alcohol-dependent patients with at least a high drinking risk level: results from a subgroup analysis of two randomized controlled 6-month studies. Alcohol and Alcoholism, 48, 570-578. doi:10.1093/alcalc/agt061.

van den Brink, W., Sørensen, P., Torup, L., Mann, K., Gual, A. y SENSE Study Group. (2014). Long-term efficacy, tolerability and safety of nalmefene as-needed in patients with alcohol dependence: A 1-year, randomised controlled study. Journal of Psychopharmacology, 28, 733-744. doi:10.1177/0269881114527362.

Whiteford, H. A., Degenhardt, L., Rehm, J., Baxter, A. J., Ferrari, A. J., Erskine, H. E.,... Vos, T. (2013). Global burden of disease attributable to mental and substance use disorders: findings from the Global Burden of Disease Study 2010. Lancet, 382, 1575-1586. doi:10.1016/ S0140-6736(13)61611-6. 